\title{
Photomyogenic response in Niemann-Pick type C: a case report
}

\author{
A. Bour $\cdot$ J. Nicolai $\cdot$ H. van Schrojenstein Lantman-de \\ Valk $\cdot$ V. van Kranen-Mastenbroek
}

Received: 12 April 2010/Revised: 12 September 2010/Accepted: 29 September 2010/Published online: 27 October 2010

(C) The Author(s) 2010. This article is published with open access at Springerlink.com

Dear Sirs,

Niemann-Pick disease type C (NP-C) is a rare autosomal recessive disorder with a wide clinical neuropsychiatric spectrum resulting from a disorder of intracellular cholesterol trafficking and synthesis caused by mutations in the $N P C 1$ or $N P C 2$ gene [1,2]. We describe a 51-year-old man with a long disease course who showed a remarkable photomyogenic (photomyoclonic) response at photic flash stimulation during EEG recordings.

Thirty years after the initial presentation of symptoms, the patient was referred for the evaluation of an early onset dementia, progressive behavioural disturbances, tremors, and gait disturbances. Until puberty a normal intelligence and psychomotor development were reported. After puberty he gradually deteriorated in daily functioning and intelligence, and at the age of 46 his IQ score was below 70 . He increasingly presented action tremors, gait disturbances,

\footnotetext{
A. Bour $(\bowtie)$

Department of Clinical Neurophysiology, Maastricht University Medical Center, P.O. Box 5800, 6202 AZ Maastricht,

The Netherlands

e-mail: A.Bour@mumc.nl

J. Nicolai

Department of Neurology, Maastricht University Medical

Center, Maastricht, The Netherlands

H. van Schrojenstein Lantman-de Valk

Department of Primary Care and Community Care, Radboud

University Medical Centre, Nijmegen, The Netherlands

H. van Schrojenstein Lantman-de Valk

Stichting Pergamijn, Echt, The Netherlands

V. van Kranen-Mastenbroek

Department of Clinical Neurophysiology, Maastricht University

Medical Center, Maastricht, The Netherlands
}

falls, myoclonic jerks in the limbs and the body, and an increased startle response. He showed severe obsessive traits and a sexual preoccupation towards young girls and women for which he was transferred to a psychiatric hospital. Neurological examination revealed poor facial mimics with a staring gaze. Speech was dysarthric and he had an aphasia. There were myoclonic jerks which increased on motor action and startle. Motor exam showed a severe ataxia of the extremities and muscle tendon reflexes were increased, but not pathologic.

Until that moment, laboratory tests, genetic counselling, and imaging studies never revealed a diagnosis. Brain MRI studies showed diffuse cerebral atrophy, most severely of the frontal and temporal lobes. Consecutive EEG recordings showed a background pattern of $8-9 \mathrm{~Hz}$ which slowed towards $7-8 \mathrm{~Hz}$. These recordings increasingly showed repetitive runs of bilateral synchronous theta activity of 4-6 Hz frontotemporally, which lasted 1-3 s. Photic flash stimulation provoked a photomyogenic response (Fig. 1). Stimulation with repetitive flashes caused time-locked muscle artefacts at the rate of flashing with increasing amplitude on faster frequencies and a sudden ending on the cessation of flash stimulation. The muscle activation bursts started $48 \mathrm{~ms}$ after the flash and had a duration of approximately $150-200 \mathrm{~ms}$. The posterior regions showed a normal photic driving response. NP-C was diagnosed when the accumulation of unesterified cholesterol by positive fibroblast Filipin staining and reduced LDL cholesterol esterification in fibroblasts was demonstrated.

At the age of 53 he succumbed from an aspiration pneumonia.

Myoclonic contractions are not a typical feature of NP-C and a photomyogenic reaction has been described only once before in NP-C [3]. Two cases of cortical myoclonus and one case on a progressive cortical action myoclonus on 

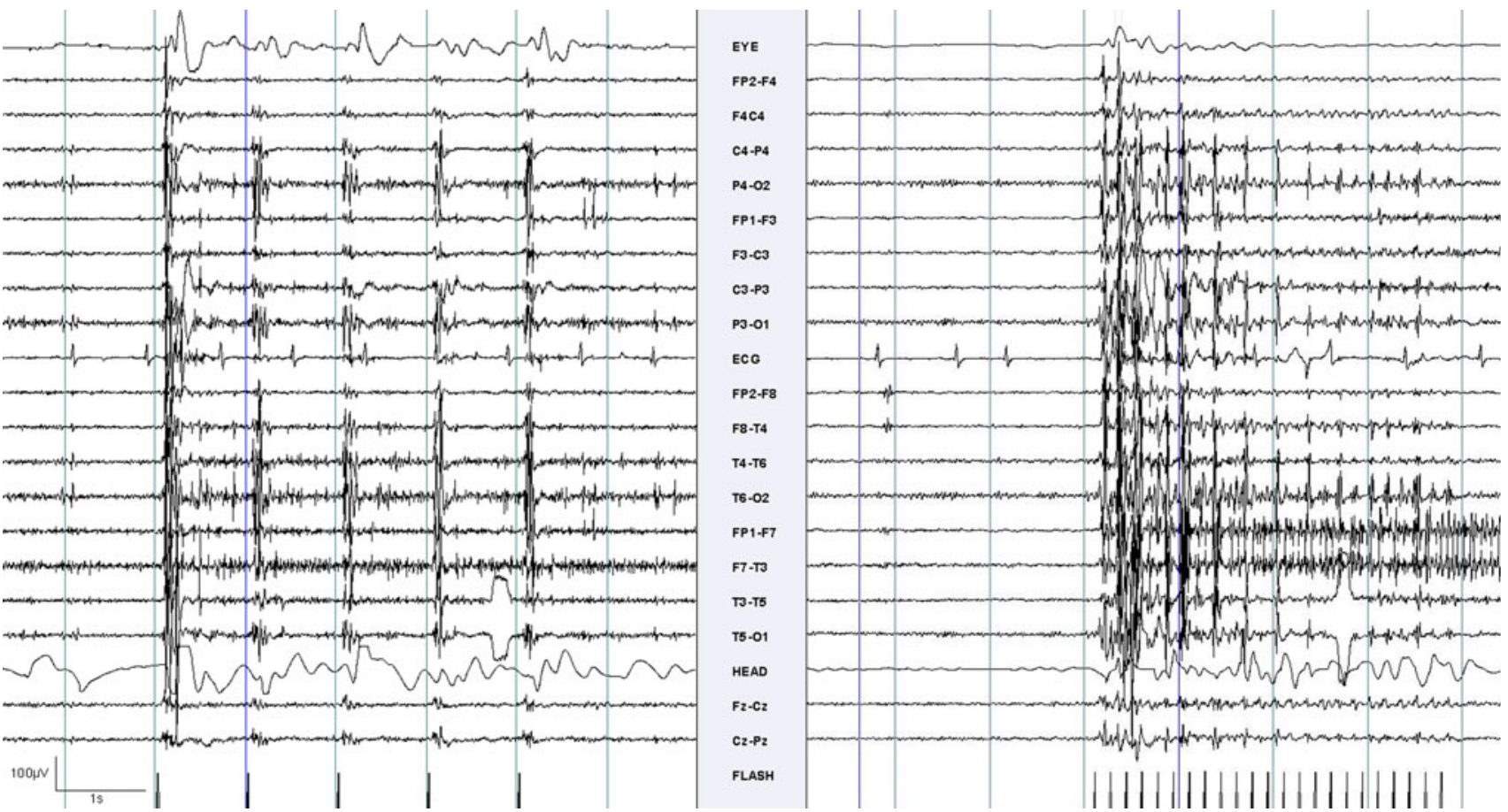

Fig. 1 EEG recording during 1 and $6 \mathrm{~Hz}$ photic flash stimulation. Rhythmic muscle activity appears in phase with the repetitive light flashes and ends at the moment photic flash stimulation is stopped

motor activity have been described $[4,5]$. The photomyogenic response results from stimulus time-locked rhythmic contractions of periocular and facial muscles in phase with the flash stimulus frequency which can extend to myoclonic jerking of the muscles of the upper body. It ceases directly after flash stimuli are stopped. It is considered to be a normal physiological response which can, however, expand under emotional circumstances or metabolic or toxic states. In our patient, EMG activity was mostly dominant in the posterior regions. Its physiological mechanism was originally thought to be of muscular origin. Myoclonus can have a cortical origin or it can be generated by the brain stem showing mechanisms similar to the blink reflex or in the reticular formation. A startle reflex induces a facial muscle reaction comparable to the blink reflex with a latency shorter than the latency we measured. Myoclonic responses generated by the reticular formation typically induce a motor reflex of the orbucularis oculi muscle $40 \mathrm{~ms}$ after the stimulus. These EMG bursts typically show a rostro-caudal recruitment starting with activiation of the cranial nerves-innervated muscles and traversing downwards to the muscles of the arms and legs [6-9]. We did not dispose of more extensive EMG recordings covering muscles of face, neck, and limbs, so we cannot be conclusive about the generator mechanism. However, EMG activity bursts in our patient were of a long duration of 150-200 ms. Moreover, the reticular reflex myoclonus is a response typically induced by touch, noise, tendon reflex, or muscle stretch. More recently, research findings suggest a cortical origin of the photomyogenic reaction, which, however, remains to be elucidated [9-11]. Myoclonic disorders have repeatedly been associated with cortical hyperexcitability $[10,12,13]$. Such a mechanism is, for instance, the case in the progressive myoclonic epilepsies. This is a progressive neurodegenerative disease characterized by seizures which can be provoked by stimuli and myoclonus of variable morphology. These patients show exaggerated evoked potentials after somatosensory and light stimuli as a result of cortical hyperexcitability. This may also occur in NP-C cases as described in this report. However, in our patient, evoked potential studies were not performed. Our patient did not have seizures, making the clinical state of our patient more consistent with a progressive myoclonic ataxia.

In the literature the neurophysiological findings of NP-C are described in only a number of reports $[2,4,5]$. EEG features reported consist of unspecific diffuse slowing of background activity. Epilepsy is described sporadically [2]. The recognition of vertical gaze palsy by electro-oculography has been described [14]. Neurophysiologic investigations may help to better understand the pathophysiological mechanism of NP-C with respect to the nervous system, may help to reveal a diagnosis, and may help to evaluate the effects of treatment. 
Open Access This article is distributed under the terms of the Creative Commons Attribution Noncommercial License which permits any noncommercial use, distribution, and reproduction in any medium, provided the original author(s) and source are credited.

\section{References}

1. Sevin M, Lesca G, Baumann N et al (2007) The adult form of Niemann-Pick disease type C. Brain 130(Pt 1):120-133

2. Higgins JJ, Patterson MC, Dambrosia JM et al (1992) A clinical staging classification for type C Niemann-Pick disease. Neurology 42(12):2286-2290

3. Josephs KA, Van Gerpen MW, Van Gerpen JA (2003) Adult onset Niemann-Pick disease type C presenting with psychosis. J Neurol Neurosurg Psychiatry 74(4):528-529

4. Floyd AG, Yu QP, Piboolnurak P, Wraith E, Patterson MC, Pullman SL (2007) Kinematic analysis of motor dysfunction in Niemann-Pick type C. Clin Neurophysiol 118(5):1010-1018

5. Canafoglia L, Bugiani M, Dalla Bernardina B et al (2006) Rhythmic cortical myoclonus in Niemann-Pick disease type C. Mov Disord 21(9):1453-1456
6. Meinck H-M (2006) Startle and its disorders. Neurophysiol Clin 36:357-364

7. Cassim F, Houdayer E (2006) Neurophysiology of myoclonus. Neurophysiol clin 36:281-291

8. Hallet M, Chadwick D, Adam J, Marsden J (1977) Reticular reflex myoclonus: a physiological type of human post-hypoxic myoclonus. J Neurol Neurosurg Psychiatry 40:253-264

9. Kanouchi T, Yokota T, Kamata T, Ishii K, Senda M (1997) Central pathway of photic reflex myoclonus. J Neurol Neurosurg Psychiatry 62(4):414-417

10. Shibasaki H (2006) Neurophysiological classification of myoclonus. Neurophysiol Clin 36(5-6):267-269

11. Artieda J, Obeso JA (1993) The pathophysiology and pharmacology of photic cortical reflex myoclonus. Ann Neurol 34(2): 175-184

12. Shibasaki H, Neshige R (1987) Photic cortical reflex myoclonus. Ann Neurol 22(2):252-257

13. Brown P, Farmer SF, Halliday DM, Marsden J, Rosenberg JR (1999) Coherent cortical and muscle discharge in cortical myoclonus. Brain 122(Pt 3):461-472

14. Garbutt, Harris CM (2000) Abnormal vertical optokinetic nystagmus in infants and children. Br J Ophthalmol 84(5):451-455 